\title{
Spinocerebellar Ataxia Type 1 protein Ataxin-1 is signalled to DNA damage by Ataxia Telangiectasia Mutated kinase
}

\author{
Celeste Suart ${ }^{1}$, Alma M. Perez ${ }^{2}$, Ismael Al-Ramahi ${ }^{2}$, Tamara Maiuri ${ }^{1}$, Juan Botas ${ }^{2}$, and Ray \\ Truant $^{1 *}$ \\ ${ }^{1}$ Department of Biochemistry and Biomedical Sciences, McMaster University, Hamilton, Ontario, Canada \\ ${ }^{2}$ Department of Molecular and Human Genetics, Department of Molecular and Cellular Biology, Jan and Dan \\ Duncan Neurological Research Institute, Texas Children's Hospital, Houston, Texas, USA
}

*To whom correspondence should be addressed: truantr@mcmaster.ca

Spinocerebellar ataxia type 1 and Huntington's disease are two CAG DNA expansion neurodegenerative diseases that have common disease modifier genes in the DNA damage response (DDR) pathway. We demonstrate that ataxin-1 localizes to sites of DNA damage in an ataxia telangiectasia mutated (ATM) kinase dependent manner and have identified the ATM substrate site in ataxin-1, thus defining a common mechanistic pathway in DDR between three age-onset neurodegenerative diseases.

\section{Spinocerebellar Ataxia Type 1} (SCA1) is an age-onset neurodegenerative disorder caused by expanded CAG DNA triplet repeats within $A T X N 1^{1}$. This expansion encodes an expanded polyglutamine tract within the ataxin-1 protein, leading to neurodegeneration within the cerebellum and brainstem ${ }^{2}$. SCA1 is a member of the age-onset polyglutamine expansion disease family, which includes Huntington's Disease (HD) ${ }^{3}$. Recent genetic correlational studies found that variations in DNA repair genes significantly modified age at onset for SCA1 and $\mathrm{HD}^{4,5}$.

We have previously characterized the response of the HD protein huntingtin to DNA damage ${ }^{6}$. Huntingtin localizes to sites of DNA damage ${ }^{6}$. This response is dependent on ataxia-telangiectasia mutated (ATM), a serine/threonine kinase activated by oxidative stress, chromatin reorganization, and DNA breaks ${ }^{7-9}$. The genetic studies indicating HD and SCA1 may share a common pathogenic mechanism involving DNA repair ${ }^{4,5}$ led us to hypothesize that ataxin-1 may have a similar response to DNA damage.

To determine if endogenous ataxin-1 can localize to sites of DNA damage, immunofluorescence was performed on hTERT-immortalized retinal pigment epithelial (RPE1) cells following irradiation of regions of interest with a 405nm laser. Post-irradiation, endogenous ataxin-1 was observed to co-localize with huntingtin at the irradiated area (Fig. 1a).

Next, we asked whether increased polyglutamine tract length beyond the pathogenic threshold of 37-39 repeats would impact ataxin-1 localization to sites of DNA damage. Polyglutamine tract length above 37-39 repeats causes SCA ${ }^{10}$. To explore the effects of these mutations, we used an eGFPfused full length ataxin-1 construct that we have previously characterized ${ }^{11}$. Live cell imaging following micro-irradiation was conducted on RPE1 cells expressing each 
eGFP-ataxin-1 construct (Fig. 1b). Similar to endogenous ataxin-1, wild type ataxin-1eGFP puncta relocated to regions of DNA damage (Fig. 1b,c). EGFP-ataxin-1 [Q84], with an expanded polyglutamine tract, had impaired relocation compared to wild type ataxin-1 [Q26] after 40 minutes (Fig. 1c). Thus, the pathogenic polyglutamine expansion affected the ability of ataxin-1 nuclear puncta to relocate to regions of damaged DNA.

We examined if ataxin-1 was recruited to chromatin following treatment with $100 \mathrm{mM}$ potassium bromate $\left(\mathrm{KBrO}_{3}\right)$, an oxidizing agent which induces DNA base damage $^{12,13}$. Protein chromatin retention assays were performed on RPE1 cells transfected with eGFP-ataxin-1 constructs and histone H2B-mCherry transfection efficiency control. Both ataxin-1 constructs displayed recruitment of ataxin-1 to chromatin in response to oxidative stress (Fig. 1d). Consistent with micro-irradiation experiments, wild type eGFP-ataxin-1 had the highest level of chromatin retention, while polyglutamine expanded eGFP-ataxin1 displayed impaired retention compared following oxidative stress (Fig. 1d). Thus, ataxin-1 chromatin association is induced by multiple types of damaging agents, and polyglutamine expansion impairs this recruitment.

We then investigated if the ataxin-1 response to DNA damage was dependent on ataxia telangiectasia mutated (ATM) kinase activity, similar to huntingtin ${ }^{6}$. We performed micro-irradiation assays on RPE1 cells expressing wild type ataxin-1 in the presence of $10 \mu \mathrm{M}$ KU55933, an ATM kinase inhibitor $^{6,14}$. Irradiated cells treated with ATM inhibitor exhibited a sharp decrease in overall cell fluorescence, as well as a decrease in the number of fluorescent nuclear puncta (Fig. 2a). There was no significant recruitment of ataxin-1 to sites of DNA damage in cells treated with ATM inhibitor (Fig. 2b). This suggested that ATM may directly phosphorylate ataxin-1.

We used the kinase prediction software GPS $3.0^{16}$ to determine if ataxin-1 contains an LSQ motif, a consensus substrate recognition motif for $\mathrm{ATM}^{15,16}$. A putative LSQ motif was identified at serine 188 in ataxin-1. This site is conserved in ataxin-1 across species and shares homology with canonical LSQ motifs (Fig. 2c). This suggested that ATM could potentially phosphorylate serine 188 in ataxin-1. A conserved serine at position 186 was also identified in our analysis (Fig. 2c). We hypothesized it might play a priming role for serine 188, similar to ATM substrate $\mathrm{p}^{2} 5^{16}$. Thus, we considered both serine 186 and 188 modification in our subsequent analyses.

To test whether phosphorylation of serine 186 or 188 affected ataxin-1 localization to DNA damage sites, serine to alanine substitution mutants were generated. RPE1 cells transfected with eGFP-ataxin-1 [Q26] S186A or eGFP-ataxin-1 [Q26] S188A substitutions were subjected to laser DNA damage assays (Fig. 2d). Despite similar expression levels, there was no recruitment of either alanine mutant to sites of DNA damage. In chromatin retention assays, both S186A and S188A ataxin-1 mutants had impaired recruitment to sites of damage compared to wild type ataxin-1 following oxidative stress (Fig 2e). This suggests that the two predicted ATM substrate residues are involved in regulating ataxin-1 localization to sites of DNA damage.

Based on our results, we generated an affinity purified rabbit polyclonal pS186pS188 ataxin-1 antibody. We then validated the antibody (see supplemental data). 
We next examined whether modulation of ATM activity affects phosphorylation levels of endogenous ataxin1 using the pS186pS188 antibody. Upon treatment with $\mathrm{KBrO}_{3}$, there was a significant increase in pS186pS188 ataxin-1 levels (Fig. 2f), indicating that this kinase substrate site was responsive to oxidative stress. Cells exposed to oxidative stress in the presence of ATM inhibitor exhibited a diminished response (Fig. 2f). Thus, oxidative stress, which increases ATM activity ${ }^{17}$, led to increased pS186pS188 ataxin-1, while inhibition of ATM activity led to decreased pS186pS188 ataxin-1. This is consistent with DNA repair factors signaled by $\mathrm{ATM}^{18}$.

Next, we investigated the levels of pS186pS188 ataxin-1 in wild type versus SCA1 human patient-derived fibroblasts immortalized by hTERT, termed TruSCA1. This is a cell model system with the highest potential genetic accuracy to human SCA1 disease with a control line not affected by SCA1 and avoiding transformation that affects TP53 pathways. TruSCA1 line was derived from primary fibroblasts from a 29 year old affected male with onset of olivopontocerebellar atrophy type I and spinocerebellar ataxia due to a CAG DNA expansion in ATXN1 of 52 repeats on one allele. Control (TruHD-Q21Q18F) and SCA1 (TruSCA1-Q52Q29M) fibroblasts were treated with $\mathrm{HBSS}$ or $100 \mathrm{mM} \mathrm{KBrO}_{3}$ for 30 minutes. There was no significant difference in pS186pS188 ataxin-1 immunofluorescent signal with or without the polyglutamine expansion (Fig. 2g). Similarly, pS186pS188 ataxin-1 levels increased significantly following $\mathrm{KBrO}_{3}$ treatment compared to control for both wild type and SCA1 fibroblasts (Fig. 2g). This indicates that human endogenous ataxin-1 pS186pS188 levels increase in response to oxidative stress, but this signaling is not affected by polyglutamine expansion.
To validate our observations in vivo, and to assess the effect of reduced ATM function on ATXN1[82Q]-induced nervous system dysfunction, we used a wellcharacterized Drosophila SCA1 model ${ }^{18}$. Nervous system-specific expression of ATXN1[82Q] (using the nrv2-GAL4 driver) leads to progressive motor performance deficits that can be quantified using movement metrics such as speed in a vial climbing assay. At 32 days of age, ATXN1[82Q] animals are 5 times slower than healthy controls in climbing assay (Fig. $3 \mathrm{a}$ and $\mathrm{b}$, compare grey lines with blue lines in b). Reduced function of the ATM Drosophila homolog, tefu, using either an inducible shRNA in the nervous system, or a heterozygous loss of function mutant, robustly ameliorates the ATXN1[82Q]induced motor deficits (Fig. 3 a and b, compare grey lines with red lines in b). These data indicate that partial reduction of tefu levels protects from ATXN1[82Q]-induced spinocerebellar ataxia in a Drosophila model of SCA1.

In this study, we describe the localization of ataxin-1 to sites of DNA damage and that this response is dependent on ATM kinase activity. Additionally, we discovered an ATM substrate site within ataxin-1 and provide evidence that ATM phosphorylates ataxin-1 at serine 188 . Finally, we show that reducing levels of the Drosophila ATM homolog completely restores motor performance in a SCA1 fly model.

The data suggests that ATM signaling DNA damage repair via ataxin-1 is not affected by the SCA1 polyglutamine expansion. However, we observed a slower dynamic response of mutant ataxin-1 puncta to the regions of DNA suggesting impeded mutant ataxin-1 dynamics may result in poorer DNA damage repair. This is consistent with $\mathrm{HD}$ and SCA1 common 
modifier genes involved in DNA damage repair.

With the identification of the ataxin-1 response to DNA damage, SCA1 joins many other forms of ataxia with pathogenic mechanisms linked to DNA repair ${ }^{19,20}$. This includes ataxia with ocular apraxia type 1 (AOA1), AOA2, Spinocerebellar ataxia with axonal neuropathy, and ataxia oculomotor apraxia XRCC1 ${ }^{21}$. Dysfunction of ATM itself causes a form of recessive ataxia ${ }^{22}$.

Overall, present findings that heterozygous loss-of-function of ATM homolog tefu or knockdown restores motor performance in a SCA1 fly model supports pursuing ATM kinase or protein expression inhibition as a potential therapeutic target for SCA1. Future work is needed to validate whether the target is enzymatic modulation of ATM kinase, or lowering ATM protein levels, as this DNA repair factor is both an enzyme and a protein scaffold. This will lead to a better understanding of SCA1 pathology and open new avenues to developing potential therapeutics that may impact a broad range of genetic age-onset neurodegenerative diseases.

\section{MATERIALS AND METHODS Reagents}

All reagents were sourced from SigmaAldrich, unless otherwise specified.

\section{Antibodies}

The antibodies against phosphorylated serines 13 and 16 of the huntingtin N17 domain were previously characterised and validated $^{23}$. Anti-ataxin-1 antibodies were from Santa Cruz Biotechnology (sc-8766). Anti-rabbit IgG conjugated to Alexa Fluor 594 and anti-goat IgG conjugated to Alexa Fluor 488 were from ThermoFisher Scientific. For the phospho-specific antibody generation; antibody was raised in New Zealand white rabbits to $\mathrm{NH}_{3}-$
G(p)SL(p)SQTPG-COOH, counter-purified over a non-phosphorylated peptide column then affinity purified over the phospho peptide column by a service from New England Peptides (MA, USA). See supplementary methods for details.

\section{Cells}

Human retinal pigmented epithelial cells immortalized with hTERT (RPE1) were from the American Type Culture Collection. RPE1 cells were cultured in DMEM/F-12 1:1 media supplemented with $10 \%$ fetal bovine serum and $0.26 \% \mathrm{NaHCO}_{3}$ at $37^{\circ} \mathrm{C}$ in a $5 \% \mathrm{CO}_{2}$ incubator under nitrogen control of oxygen levels to $4 \%$.

TruHD-Q21Q18F wild type cells were generated and cultured as described previously $^{24}$. TruSCA1-Q52Q29M hTERT immortalized fibroblasts were generated as follows: SCA1 patient fibroblasts were purchased from the Coriell Institute repository (GM06927). Cells were cultured in MEM with 15\% fetal bovine serum and $1 \mathrm{X}$ GlutaMAX (Life Technologies \#35050). Cells were infected with $1 \times 10^{6}$ TERT Human Lentifect Purified Lentiviral Particles (GeneCopoeia, LPP-Q0450-Lv05-200-S). To aid in infection, $10 \mu \mathrm{g} / \mathrm{ml}$ polybrene was added. After 8 h, cells were infected again and left for $24 \mathrm{~h}$. Media was changed, and cells were left for an additional $48 \mathrm{~h}$. Successfully transduced cells were selected in media with $1 \mu \mathrm{g} / \mathrm{ml}$ puromycin. Cells were grown at $37^{\circ} \mathrm{C}$ with $5 \% \mathrm{CO}_{2}$ and $4 \%$ oxygen levels.

\section{Transfections}

All cells were transfected with TransIT-X2 (Mirus Bio) according to the manufacturer's specifications. All transfections involving ataxin-1 constructs were incubated at $37^{\circ} \mathrm{C}$ for 8 hours with $1 \mu \mathrm{g}$ of DNA. Ataxin-1 expression plasmids; eGFP-ataxin-1 [Q26], eGFP-ataxin-1 [Q84]; were generated as described previously ${ }^{11}$. EGFP-ataxin-1 [Q26] 
S186A and eGFP-ataxin-1 [Q26] S188A were generated from eGFP-ataxin-1 [Q26] using Q5 Site-Directed Mutagenesis Kit according to the manufacturer's specifications. All PCR reagents and enzymes were purchased from New England Biolabs. Plasmids were purified via Presto Mini Plasmid Kit (Geneaid) and sequences were verified by PCR sequencing by the McMaster Mobix facility.

\section{Immunofluorescence}

Cells were fixed using methanol at $-20^{\circ} \mathrm{C}$ for 20 minutes, then washed with wash buffer (50mM Tris- $\mathrm{HCl}, \mathrm{pH} 7.5,150 \mathrm{mM} \mathrm{NaCl}$, $0.1 \%$ Triton X-100). Then, cells were blocked with blocking buffer (wash buffer + 5\% FBS) for either 10 minutes at room temperature or overnight at $4^{\circ} \mathrm{C}$. Cells were incubated with primary antibodies diluted in blocking buffer for 1 hour at room temperature. Next, cells were washed and then incubated with secondary antibodies diluted in blocking buffer for 20 minutes at room temperature. Following washing with wash buffer, cells were imaged in PBS.

\section{Microscopy}

All microscopy was completed using a Nikon A1 confocal system attached to a Nikon Eclipse Ti inverted microscope, using an PLAN APO 60x/1.40 oil objective or PLAN APO $20 \times / 0.75$ dry objective with Spectra $X$ LED lamp (Lumencor) and GaAsP detectors. A 405nm laser which was part of the Nikon A1 confocal system was used for irradiation experiments. 405nm, 489nm, and 561nm lasers experiments were used for imaging.

\section{Micro-irradiation Assay}

RPE1 cells were grown in glass-bottom six well tissue culture dishes or eight well $\mu$-slide ibiTreat until $80-85 \%$ confluence, then stained with NucBlue (ThermoFisher Scientific) for 15 minutes at $37^{\circ} \mathrm{C}$ in a $5 \%$ $\mathrm{CO}_{2}$ incubator. Media was aspirated and replaced with Hank's Balanced Salt Solution
(HBSS) (ThermoFisher Scientific) immediately preceding irradiation. Using the Nikon A1 confocal setup described in the subsequent section, samples were kept at $37^{\circ} \mathrm{C}$ with a Tokai Hit Inu Incubation system (model WSKM). A 405nm laser set to $100 \%$ power was used to irradiate regions of interest using a scan speed of 1/16 frames per second (512 x 512 pixels). Regions of interest were drawn over areas where ataxin-1 nuclear inclusions were absent. After X-Y coordinates were recorded, cells were either imaged live, or incubated at $37^{\circ} \mathrm{C}$ for specified incubation times preceding methanol fixations and immunofluorescence. Then $\mathrm{X}-\mathrm{Y}$ coordinates were revisited for imaging. For live cell imaging, cells transfected with ataxin-1 constructs were irradiated as described above. Cells were imaged at either 1 minute or 2 minute intervals over a thirty-minute period using confocal microscopy as described in the subsequent section. For ATM kinase inhibition trials, cells were incubated for 15 minutes in HBSS with $10 \mu \mathrm{M}$ ATM kinase inhibitor KU55933 prior to irradiation.

\section{Average Pixel Intensity Quantification} Average pixel intensity of the regions of interest were measured using ImageJ mean gray function on FITC channel image. Higher intensity indicates presence of ataxin-1 nuclear inclusions. Values were normalized to the average of pre-irradiation control cell values, which had a region of interest defined but were not irradiated. This analysis was conducted at three time points: 0 minutes, 10 minutes, 25 minutes, and 40 minutes. Statistical analysis, including Student's t-test and ANOVA, were completed using GraphPad Prism software.

\section{Chromatin Retention Assay}

RPE1 cells were transfected with indicated eGFP-ataxin-1 construct and transfection control histone 2B-mCherry (H2BmCherry). After 24 hours incubation at $37^{\circ} \mathrm{C}$, 
cells treated with either HBSS (control) or $100 \mathrm{mM} \mathrm{KBrO}_{3}$ in $\mathrm{HBSS}$ for 30 minutes. Soluble proteins were extracted with cold $0.2 \%$ Triton $\mathrm{X}-100$ in PBS for 2 minutes on ice, then cells were fixed with $4 \%$ paraformaldehyde for 15 minutes at room temperature. Nuclear intensity was quantified using CellProfiler ${ }^{25}$, using H2B mCherry to normalize for variability in transfection efficiency. Intensity was normalized to untreated wild type ataxin-1 conditions.

\section{pS186pS188 Ataxin-1 Oxidative Stress Trials}

RPE1 cells were incubated at $37^{\circ} \mathrm{C}$ for 30 minutes with either HBSS (control), $10 \mu \mathrm{M}$ KU55933 in HBSS, $100 \mathrm{mM} \mathrm{KBrO}_{3}$ in HBSS, or $10 \mu \mathrm{M}$ KU55933 and $100 \mathrm{mM}$ $\mathrm{KBrO}_{3}$ in HBSS. Cells were then fixed with methanol and immunofluorescence was conducted as described above. Raw nuclear intensity was quantified using CellProfiler, with values being normalized to the average of the HBSS control values.

\section{Drosophila Models and Motor Performance Tests}

The Drosophila SCA1 model and transgenic lines expressing human ATXN1[82Q] were previously described ${ }^{18}$. The inducible shRNA line v108074 specifically targeting Tefu, the Drosophila ATM orthologue, and the control, non-targeting, scramble shRNA v2691 were obtained from the Vienna Drosophila Stock Center. The nervous system driver line nrv2GAL4, and the Tefu (dATM) loss-of-function allele Mi\{ET1\}tefuMB09945 were obtained from the Bloomington Drosophila Stock Center. For the Drosophila motor performance assay, we used an automated data acquisition system. This system taps the vials at $7 \mathrm{~s}$ intervals and records video files. These files are then processed using a custom-built software that calculates the average speed of the animals in each vial. 10 age-matched virgin females were used per replicate, and at least four replicates per genotype were tested. Animals are transferred into vials containing fresh media daily. For statistical analysis we performed linear mixed models ANOVA between the indicated genotypes using four replicates per genotype. All cultures, breeding and tests were performed at $25^{\circ} \mathrm{C}$.

\section{Genotypes:}

negative controls: $\quad w[1118] /+, \quad$ nrv2GAL4/UAS-v2691. ATXN1[82Q]/scramble: UASATXN1[82Q]F7/w[1118]; nrv2GAL4/UAS-v2691. ATXN1[82Q]/dATMsh1: UAS-ATXN1[82Q]F7/w[1118]; nrv2GAL4/UAS-v108074.

ATXN1[82Q]/dATM+/-: $\quad$ UASATXN1[82Q]F7/w[1118]; nrv2-GAL4/+; Mi\{ET1\}tefuMB09945/+

\section{FUNDING}

Work supported by the Krembil Foundation.

\section{CONFLICT OF INTEREST STATEMENT}

None declared.

\section{ABBREVIATIONS}

AOA1 Ataxia with ocular apraxia type 1

AOA2 Ataxia with ocular apraxia type 2

ATM Ataxia-telangiectasia mutated

DDR DNA Damage repair

HBSS Hank’s balanced salt solution

HD Huntington's Disease

RPE1 Retinal pigment epithelial cells

SBMA Spinobulbar muscular atrophy

SCA1 Spinocerebellar ataxia type 1

SCA3 Spinocerebellar ataxia type 3

SCA17 Spinocerebellar ataxia type 17 


\section{LEGENDS TO FIGURES}

Figure 1. Ataxin-1 localizes to sites of DNA damage following irradiation and oxidative stress. All graphs display mean $( \pm \mathrm{SD})$. Significance values calculated by two-way ANOVA, $* * *=\mathrm{p} \leq 0.001, * * * *=\mathrm{p} \leq 0.0001$. a. Laser microirradiation assay of endogenous ataxin-1 from RPE1 cell. White rectangle indicates irradiated area. b. Laser microirradiation assay of the indicated transfected ataxin-1 constructs in RPE1 cells. White rectangle indicates irradiated area. c. Quantification of part b. Average pixel intensity was calculated for control ( $0 \%$ laser power) and irradiated (100\% laser power) regions. Values normalized to the average pre-irradiation control value to account for random movement of ataxin- 1 puncta. Both constructs had significant difference from non-irradiated controls starting at 10 minutes. d. Chromatin retention assay with indicated transfected ataxin-1 constructs. Nuclear eGFP intensity was quantified after extraction of soluble proteins. Potassium bromate treated conditions both significantly different from controls $(\mathrm{p} \leq 0.0001)$. Approximately 30 images were taken per condition with 20-40 cells er image.

Figure 2. ATM inhibition prevents ataxin-1 localization to DNA damage. a. Laser microirradiation assay of RPE1 cells transfected with eGFP-ataxin-1[Q26] after incubation with $10 \mu \mathrm{M}$ ATM kinase inhibitor KU55933. b. Values from part a were quantified as in Figure 1c. Error bars show mean ( \pm SD) on the basis of six replicates. Significance values calculated by two-way ANOVA, $* *=\mathrm{p} \leq 0.01, * * *=\mathrm{p} \leq 0.001$. c. Alignment and conservation of predicted ATM kinase site in ataxin-1. p53, PTS, and p95 are canonical ATM substrate provided for comparison. Residues 185-192 of ataxin-1 shown. Adapted from Kim et al., 1999. Serine 186 shown in red, serine 188 shown in blue. d. Quantification of indicated eGFP-ataxin-1 construct recruitment to micro-irradiated regions. Error bars and statistical tests as described in Figure 1c. eGFP-ataxin-1 [Q26] data displayed is replicated from Figure 1c for comparison. e. Chromatin retention assay of eGFP-ataxin-1 [Q26] S186A and eGFP-ataxin-1 [Q26] S188A compared to eGFP-ataxin-1 [Q26], using methods and statistical analysis as described in Figure 1d. eGFPataxin-1 [Q26] data displayed is replicated from Figure 1d for comparison. f. Oxidative stress increases pS186pS188 ataxin-1 levels in cells in an ATM-dependent manner. RPE1 cells were treated with either unsupplemented HBSS (control), $10 \mu \mathrm{M} \mathrm{KU} 55933,100 \mathrm{mM} \mathrm{KBrO}_{3}$, or $10 \mu \mathrm{M}$ KU55933 and $100 \mathrm{mM} \mathrm{KBrO}_{3}$ for 30 minutes. Quantification was completed with CellProfiler. For each condition, approximately 30 images were taken with 20-40 cells per image. Error bars displays SD. Significance values calculated by one-way ANOVA, $* *=p \leq 0.01$, **** $=\mathrm{p} \leq$ 0.0001. g. SCA1 or wild type fibroblast cells were treated with either unsupplemented HBSS (control) or $100 \mathrm{mM} \mathrm{KBrO}_{3}$ for one hour. Quantification completed as described in $\mathrm{f}$. Potassium bromate treated conditions are both significantly different from controls $(\mathrm{p} \leq 0.0001)$.

Figure 3. Knockdown of the Drosophila ATM homolog ameliorates ATXN1[82Q]-induced nervous system dysfunction. a, Time-sequence images illustrating the behavior of 24-day-old Drosophila females in a motor performance test. At 1s, all 10 age-matched virgin females of identical genotype were tapped to the bottom of a vial. After 7s, most of the negative control (healthy) fruit flies were able to climb past the midway point of the vial, whereas the majority of flies expressing ATXN1[82Q] in the nervous system (nerv-GAL4, also expressing a scramble, non-targeting shRNA) were at the bottom of the vial. ATXN1[82Q]-expressing animals in which the ATM fly homolog was knocked down specifically in the nervous system using an inducible shRNA (ATXN1[82Q]/dATM-sh), or carrying a heterozygous loss-of-function mutation in 
dATM (ATXN1[82Q]/dATM+/-), showed amelioration of ATXN1[82Q]-induced motor impairments. b, Graphs representing climbing speed as a function of age in healthy control fruit flies (blue lines), or in flies expressing ATXN1[82Q] alone (gray lines), or together with the dATM-sh or the dATM+/- loss-of-function allele (red lines). Two replicates are shown for each genotype. Error bars indicate s.e.m. The data was analyzed using linear mixed models ANOVA using a total of four independent replicates per genotype. ${ }^{*}=\mathrm{p}<0.0001$ between ATXN1[82Q] and either ATXN1[82Q]/dATM-sh or ATXN1[82Q]/dATM+/-. 


\section{FIGURES}

a

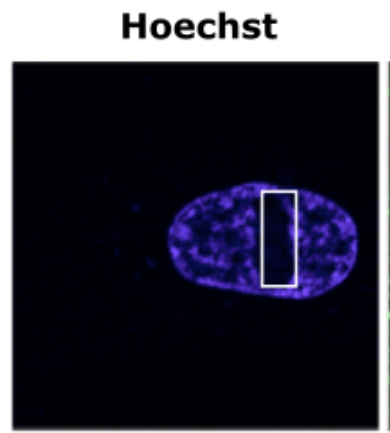

O Minutes
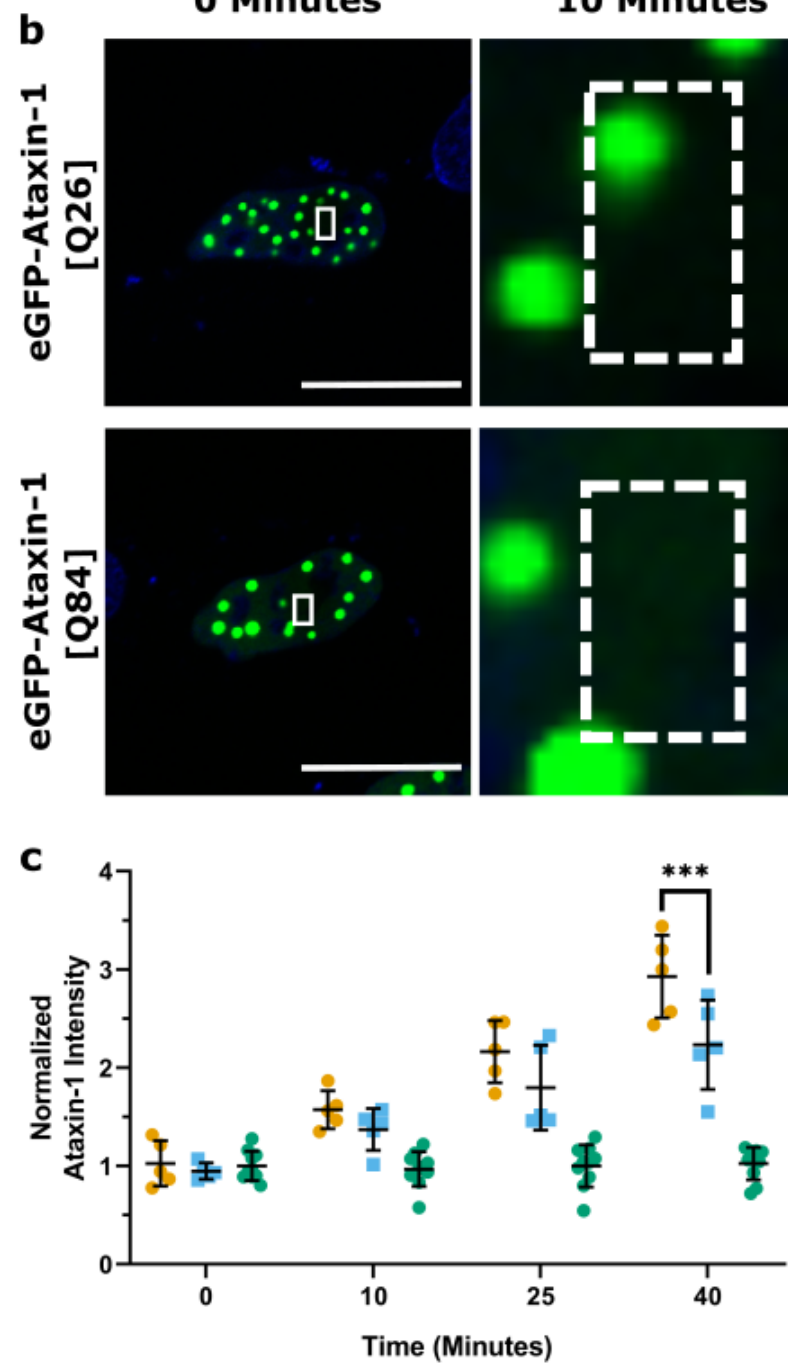

- eGFP-ataxin-1 [Q26] = eGFP-ataxin-1 [Q84]

- Non-Irradiated Controls

Ataxin-1

10 Minutes
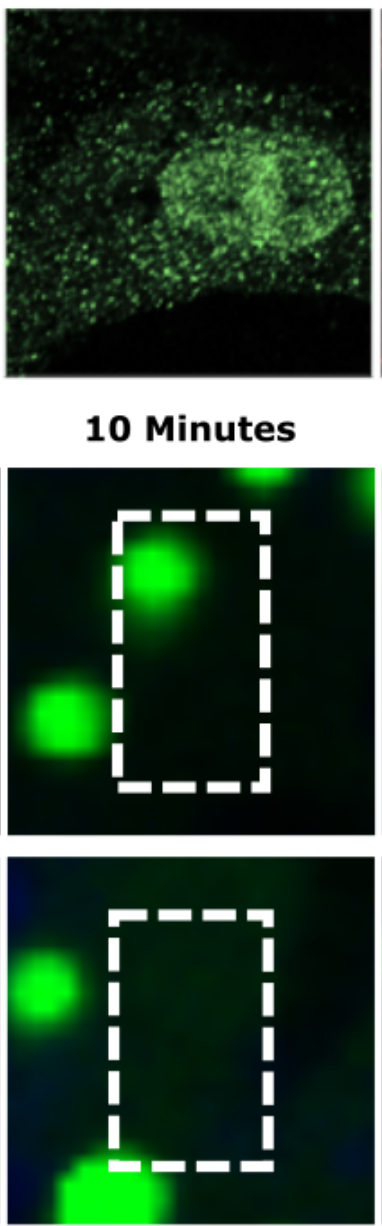

d

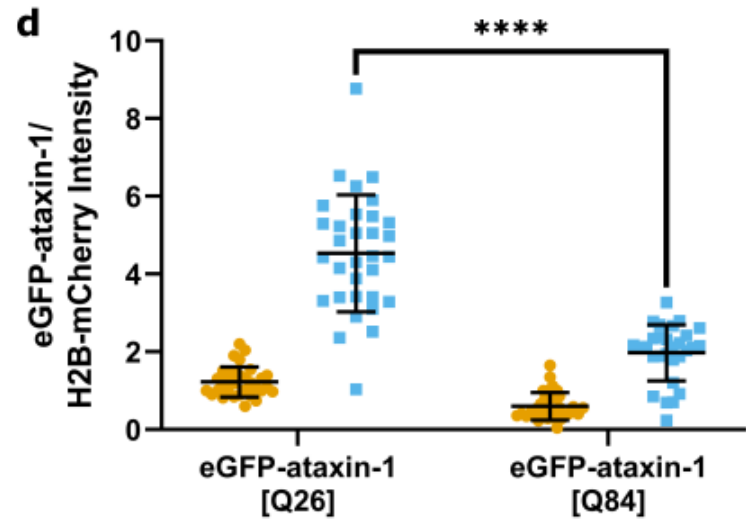

25 Minutes
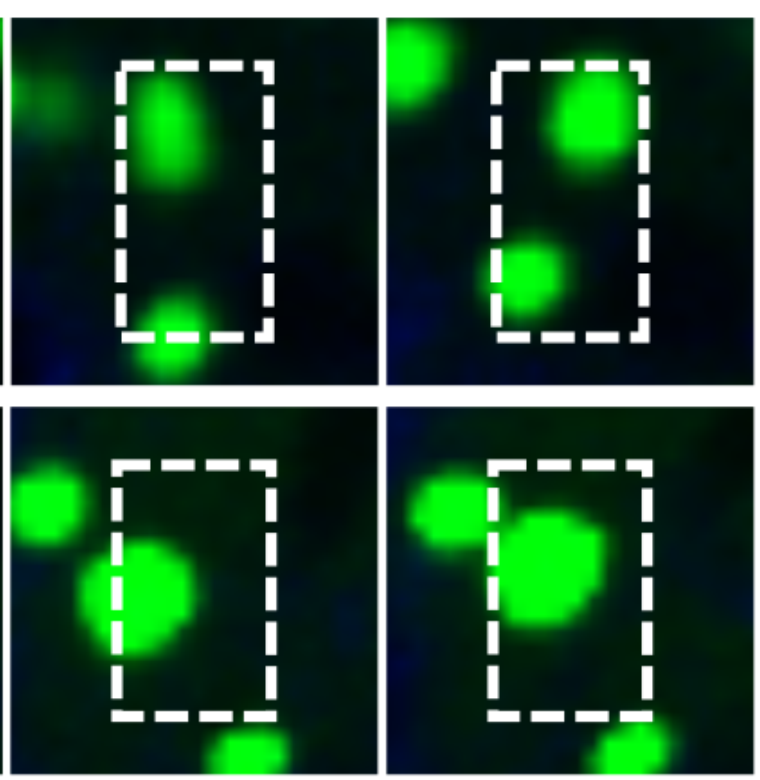

- Control = $100 \mathrm{mM} \mathrm{KBrO}_{3}$

Merge

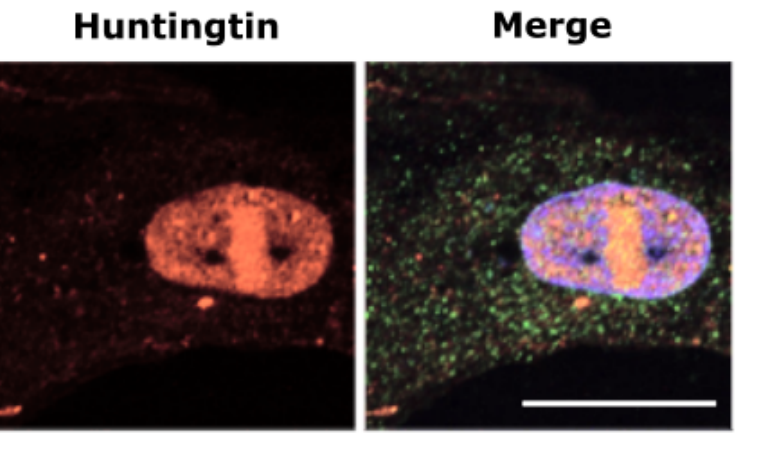

40 Minutes

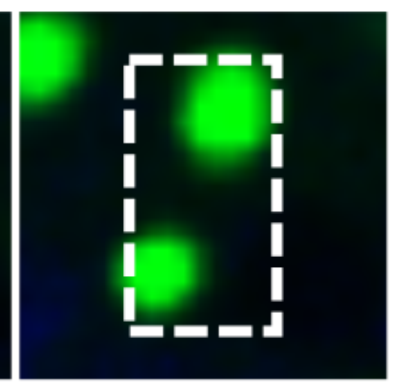

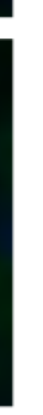


a
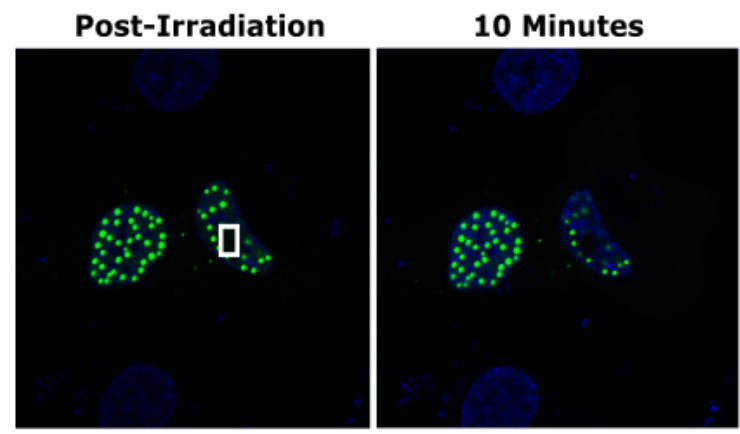

b

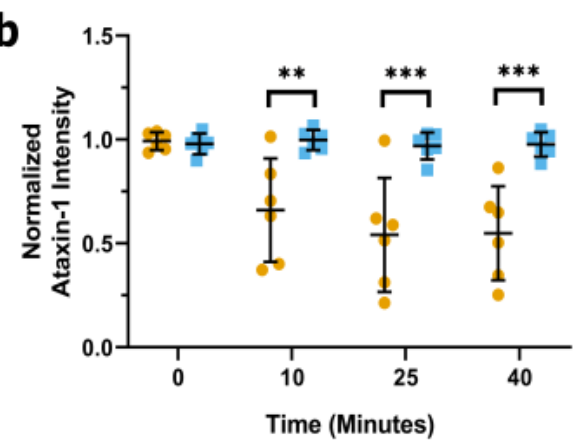

d

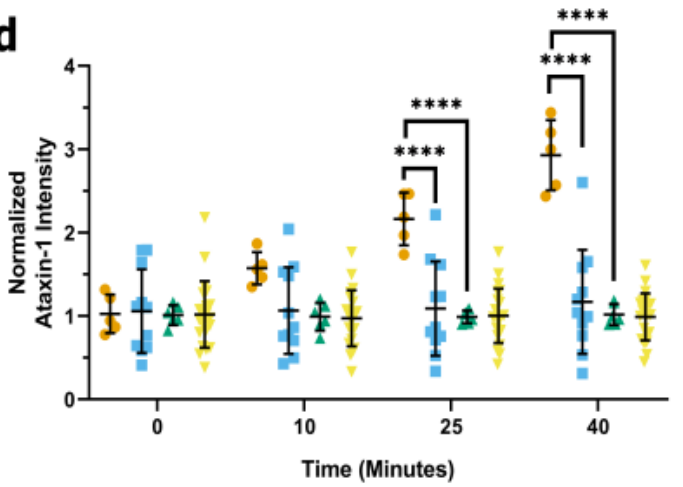

- eGFP-ataxin-1 [Q26] \ eGFP-ataxin-1 [Q26] S188A

" eGFP-ataxin-1 [Q26] S186A ₹ Non-Irradiated Controls

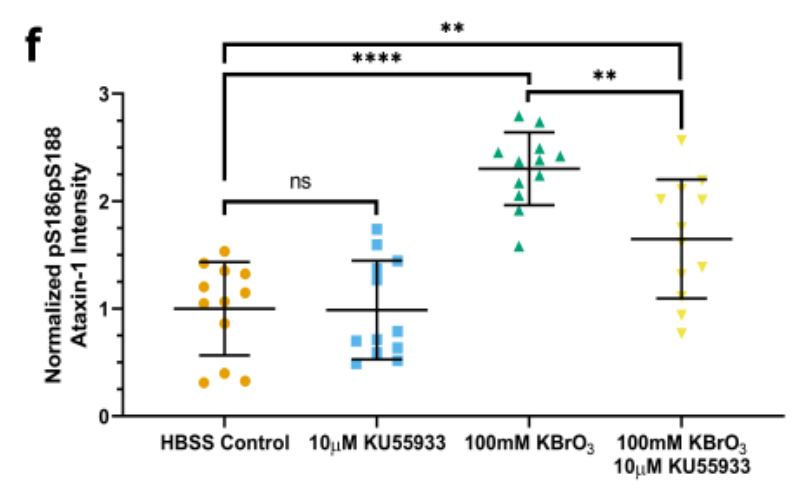

25 Minutes
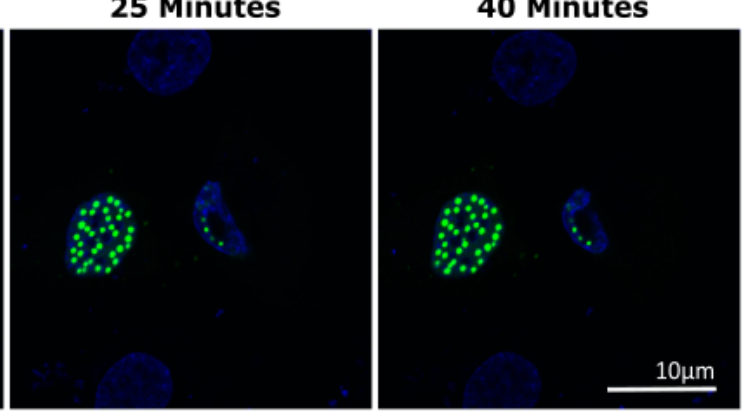

C

P53: PPLSQETF

PTS: PDLSQAEI

P95: PSLSQGVS

Ataxin-1 Human: GSLSQTPG

Ataxin-1 Mouse: GSLSQTPG

Ataxin-1 Danio: SLDSQSHV

Ataxin-1 Fugu: SPLSQSTA
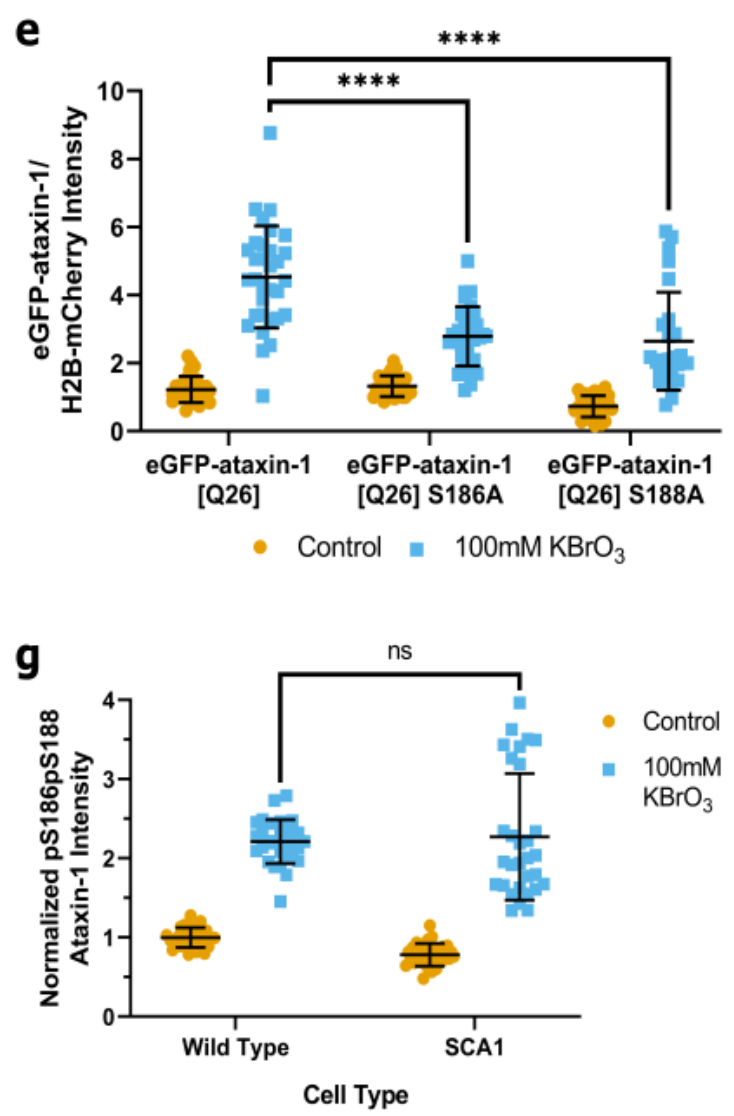

Figure 2 


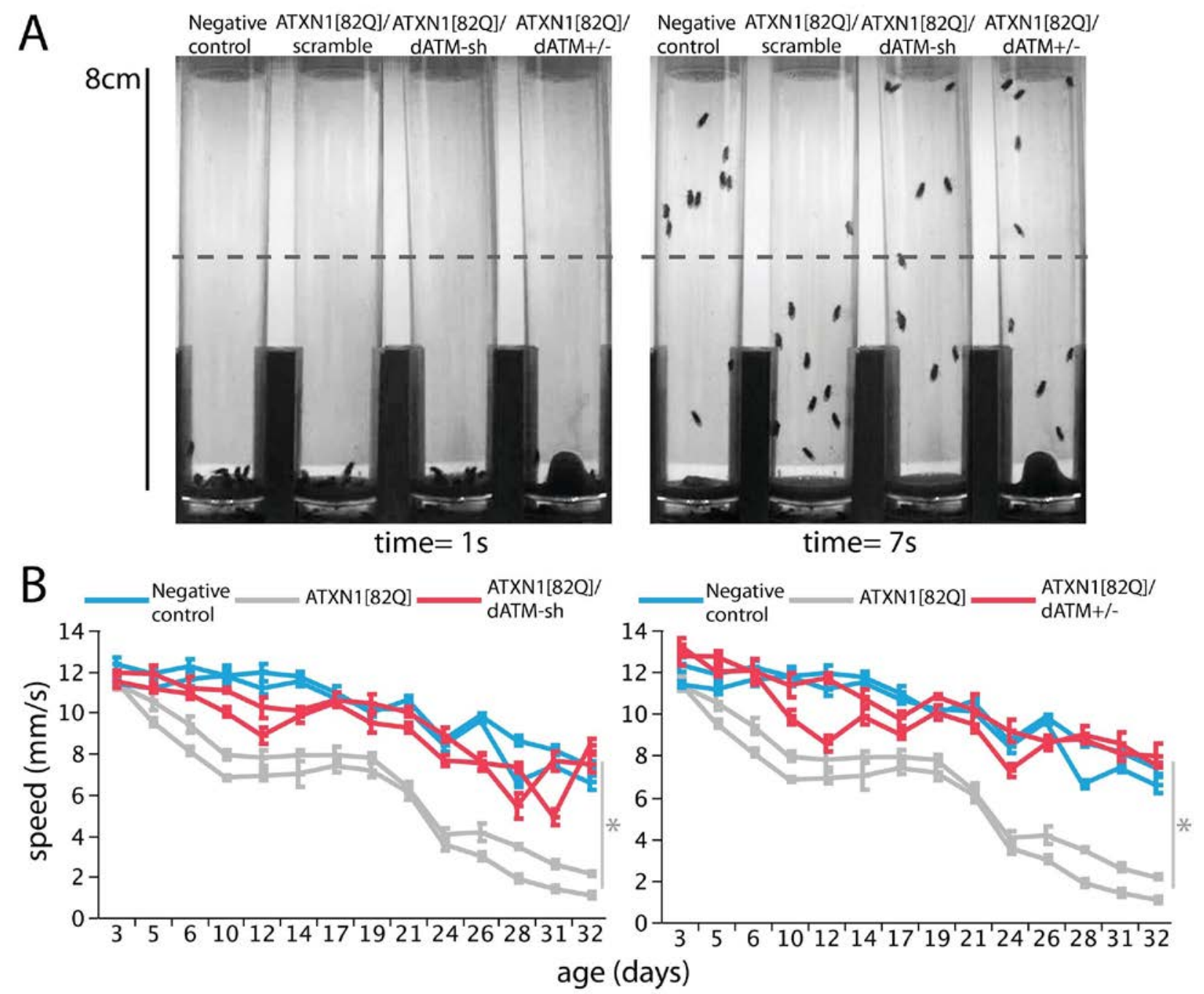

Figure 3 


\section{SUPPLEMENTARY MATERIAL}

\section{Phospho-Specific Antibody Generation}

The antibody was raised in New Zealand white rabbits to $\mathrm{NH}_{3}-\mathrm{G}(\mathrm{p}) \mathrm{SL}(\mathrm{p}) \mathrm{SQTPG}-\mathrm{COOH}$, then affinity purified over peptide column. All steps, including peptide synthesis, injections, titrations, bleeds, and affinity purification, were carried out at New England Peptide (Garner, MA, USA) with the standard PSSA Rabbit package. The antibody was then validated by dot-blot assay to determine epitope specificity; western blotting following siRNA knockdown to determine protein specificity; and peptide competition assay to determine immunofluorescent signal specificity.

\section{Dot Blot Assay}

Serial dilution concentrations (25, 50, 100, 500, 1000ng) of synthetic ataxin-1 peptides (nonphospho GSLSQTPG and phospho GPSLPSQTPG) were spotted onto nitrocellulose membrane (Pall Life Sciences) and were dried at room temperature for 45 minutes.

The membrane was blocked with 5\% non-fat milk powder in TBS-T (50 mM Tris-HCl, $\mathrm{pH} 7.5$, $150 \mathrm{mM} \mathrm{NaCl}, 0.1 \%$ Tween-20) for 1 hour at room temperature. The membrane was then incubated with primary phospho-ataxin-1 antibody (1:1000) overnight at $4^{\circ} \mathrm{C}$. The membrane was washed for 10 minutes three times with TBS-T, then incubated with anti-rabbit peroxidase (HRP) secondary (1:50,000; Abcam) for 45 minutes at room temperature. The membrane was again washed for 10 minutes three times with TBS-T, then imaged with enhanced chemiluminescent HRP substrate (EMD Millipore) on a MicroChemi system (DNR Bio-imaging Systems). The membrane was then rinsed with TBS-T and re-imaged with Ponceau stain. 
siRNA Knockdown

Endogenous ataxin-1 knockdown was established with ataxin-1 SMARTpool siGENOME siRNA (Dharmacon, M-004510-02-0005) in RPE1 cells. siRNA was transfected with Lipofectamine RNAiMax (Invitrogen) according to the manufacturer’s instructions. Control dishes were established with scrambled siRNA.

Cells were lysed in radioimmunoprecipitation assay buffer with 10\% phosphatase (Roche) and 10\% protease inhibitors (Roche), scrapped, and incubated on ice for 15 minutes. Cell lysates were centrifuged at full speed for 10 minutes at $4^{\circ} \mathrm{C}$. Protein concentration was quantified by Bradford assay, then 40-60 $\mu$ g of protein was loaded into a precast 4-20\% polyacrylamide gradient gel (Biorad). Proteins were then separated by SDS-PAGE and electroblotted onto 0.45 $\mu$ m polyvinyl difluoride (PVDF) membrane (EMD Millipore). Western immunoblotting was then conducted.

\section{Western Immunoblotting}

Immunoblots were blocked with 5\% non-fat milk powder in TBS-T (50 mM Tris-HCl, pH 7.5, $150 \mathrm{mM} \mathrm{NaCl,} \mathrm{0.1 \%} \mathrm{Tween-20)} \mathrm{for} 1$ hour at room temperature. Blots were cut horizontally at a 48-kDa marker to probe for ataxin-1 (87 kDA) or GAPDH (loading control, 37 kDa) separately. Blots were then incubated with primary phospho-ataxin-1 antibody (1:1000) or GAPDH (1:10,000; Abcam ab8425) overnight at $4^{\circ} \mathrm{C}$. Blots were washed for 10 minutes three times with TBS-T, then incubated with anti-rabbit peroxidase (HRP) secondary (1:50,000; Abcam) for 45 minutes at room temperature. Blots were again washed for 10 minutes three times with TBS-T, then imaged with enhanced chemiluminescent HRP substrate (EMD Millipore) on a MicroChemi 
system (DNR Bio-imaging Systems). Bands were quantified using National Institutes of Health ImageJ. Phospho-ataxin-1 signal was normalized to the GAPDH loading control.

Immunofluorescence Peptide Competition Assay

The phospho-ataxin-1 antibody (1:250 was incubated with 1000 ng of synthetic peptides (nonphospho ataxin-1, phospho ataxin-1, and p53 control peptide) at room temperature for 1 hour. RPE1 cells were then fixed with cold methanol as previously described, blocked with 10\% FBS in TBS $+0.1 \%$ TritX for 1 hour, then incubated overnight with the treated antibodies. Cells were washed three times with PBS, then incubated with anti-rabbit AlexaFluor488 secondary antibody (1:500, Molecular Probes) for 45 minutes at room temperature. Cells were then washed three times with PBS, then imaged as previously described.

\section{Supplemental Figures}

Figure S1. pS186pS188 ataxin-1 antibody validation. a. Dot blot assay with ataxin-1 peptide in the unphosphorylated (S186S188) and phosphorylated (pS186pS188) state. b. Ataxin-1 knockdown showing pS186pS188 antibody specificity compared to previously validated 11750 antibody. Blots were cut at the $48 \mathrm{kDa}$ marker to probe for ataxin-1 and GAPDH separately. p= 0.0063 for 11750 ataxin-1, $\mathrm{p}=0.0767$ for $\mathrm{pS186pS188}$ ataxin-1. Analyzed by unpaired t-test of three independent replicates. Error bars indicate standard deviation. c. Peptide competition assay with unphosphorylated S186S188, phosphorylated pS186pS188, and non-specific TP53 peptide. The cytoplasmic signal is non-specific. Note that in no peptide control, S186S188, and TP53 there is evenly distributed signal in the nucleus, with transient stress events causing an increase in nuclear signal for some cells. In pS186pS188, the nuclear signal is reduced and no increase in nuclear signal is observed. 


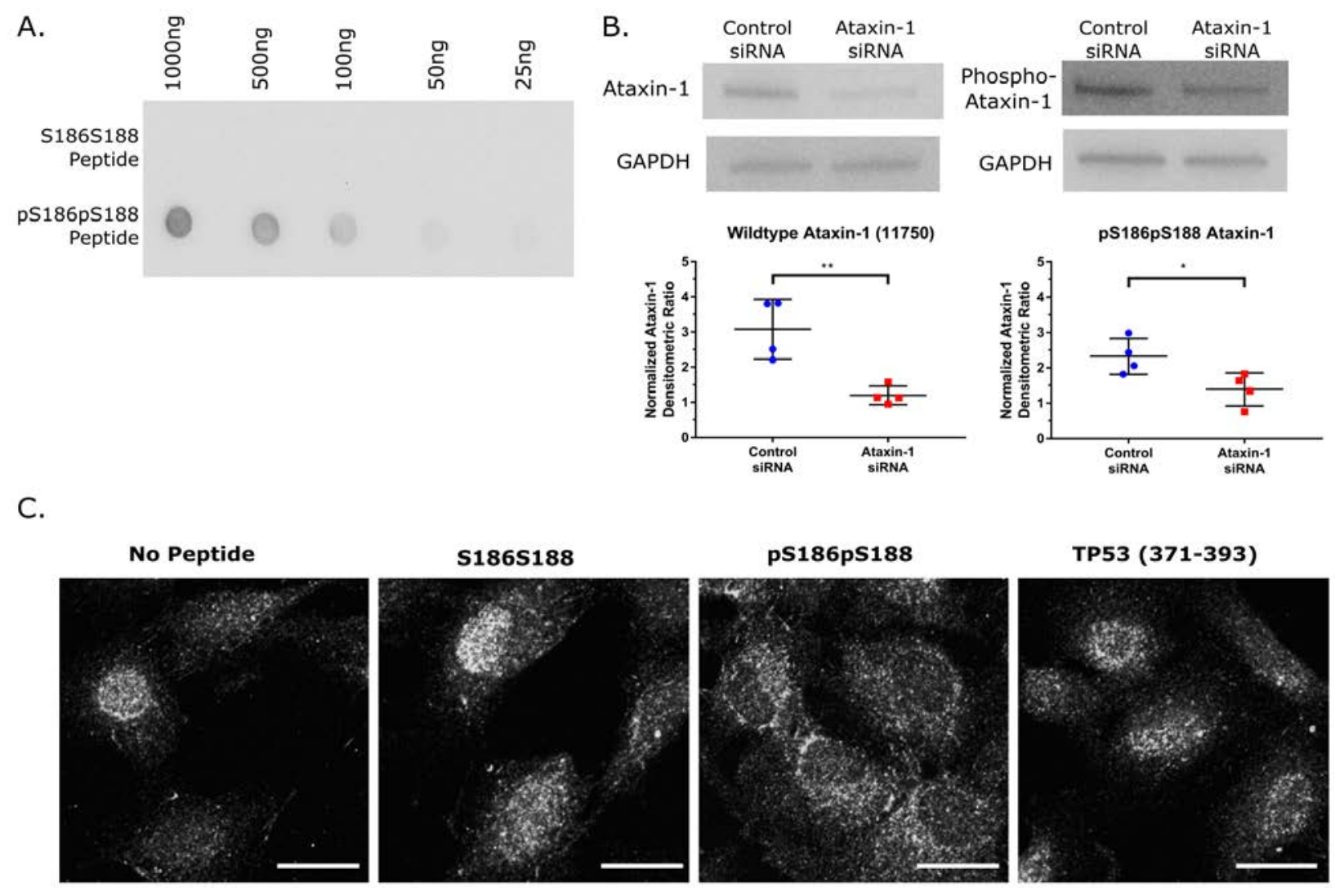

Supplemental Figure 1 


\section{REFERENCES}

1. Orr, H. T. et al. Expansion of an unstable trinucleotide CAG repeat in spinocerebellar ataxia type 1. Nat. Genet. 4, 221-226 (1993).

2. Koeppen, A. The pathogenesis of spinocerebellar ataxia. Cerebellum 4, 62-73 (2005).

3. Fan, H.-C. et al. Polyglutamine (PolyQ) Diseases: Genetics to Treatments. Cell Transplant. 23, 441458 (2014).

4. Bettencourt, C. et al. DNA repair pathways underlie a common genetic mechanism modulating onset in polyglutamine diseases. Ann. Neurol. 79, 983-990 (2016).

5. Genetic Modifiers of Huntington's Disease (GeM-HD) Consortium. Identification of Genetic Factors that Modify Clinical Onset of Huntington’s Disease. Cell 162, 516-526 (2015).

6. Maiuri, T. et al. Huntingtin is a scaffolding protein in the ATM oxidative DNA damage response complex. Hum. Mol. Genet. 26, 395-406 (2017).

7. Khoronenkova, S. V. \& Dianov, G. L. ATM prevents DSB formation by coordinating SSB repair and cell cycle progression. Proc. Natl. Acad. Sci. U. S. A. 112, 3997-4002 (2015).

8. Bakkenist, C. J. \& Kastan, M. B. DNA damage activates ATM through intermolecular autophosphorylation and dimer dissociation. Nature 421, 499-506 (2003).

9. Guo, Z., Kozlov, S., Lavin, M. F., Person, M. D. \& Paull, T. T. ATM Activation by Oxidative Stress. Science 330, 517-521 (2010).

10. Schmitz-Hübsch, T. et al. Spinocerebellar ataxia types 1, 2, 3, and 6: disease severity and nonataxia symptoms. Neurology 71, 982-989 (2008).

11. Irwin, S. et al. RNA association and nucleocytoplasmic shuttling by ataxin-1. J. Cell Sci. 118, 233242 (2005).

12. Ballmaier, D. \& Epe, B. Oxidative DNA damage induced by potassium bromate under cell-free conditions and in mammalian cells. Carcinogenesis 16, 335-342 (1995).

13. Campalans, A. et al. Interaction with OGG1 is required for efficient recruitment of XRCC1 to base 
excision repair and maintenance of genetic stability after exposure to oxidative stress. Mol. Cell. Biol. 35, 1648-1658 (2015).

14. Hickson, I. et al. Identification and characterization of a novel and specific inhibitor of the ataxiatelangiectasia mutated kinase ATM. Cancer Res. 64, 9152-9159 (2004).

15. Xue, Y. et al. GPS 2.1: enhanced prediction of kinase-specific phosphorylation sites with an algorithm of motif length selection. Protein Eng. Des. Sel. 24, 255-260 (2010).

16. Kim, S. T., Lim, D. S., Canman, C. E. \& Kastan, M. B. Substrate specificities and identification of putative substrates of ATM kinase family members. J. Biol. Chem. 274, 37538-37543 (1999).

17. Lee, S. A., Dritschilo, A. \& Jung, M. Role of ATM in oxidative stress-mediated c-Jun phosphorylation in response to ionizing radiation and CdCl2. J. Biol. Chem. 276, 11783-11790 (2001).

18. Maréchal, A. \& Zou, L. DNA damage sensing by the ATM and ATR kinases. Cold Spring Harb. Perspect. Biol. 5, (2013).

19. Gueven, N. et al. A subgroup of spinocerebellar ataxias defective in DNA damage responses. Neuroscience 145, 1418-1425 (2007).

20. Rass, U., Ahel, I. \& West, S. C. Defective DNA Repair and Neurodegenerative Disease. Cell 130, 991-1004 (2007).

21. Ross, C. A. \& Truant, R. A unifying mechanism in neurodegeneration. Nature 541, 34-35 (2017).

22. Shiloh, Y. Ataxia-telangiectasia and the Nijmegen breakage syndrome: related disorders but genes apart. Annu. Rev. Genet. 31, 635-662 (1997).

\section{SUPPLEMENTAL REFERENCES}

23. Atwal, R. S. et al. Kinase inhibitors modulate huntingtin cell localization and toxicity. Nat. Chem. Biol. 7, 453-460 (2011).

24. Hung, C. L.-K. et al. A patient-derived cellular model for Huntington’s disease reveals phenotypes at clinically relevant CAG lengths. Molecular Biology of the Cell 29, 2809-2820 (2018). 
bioRxiv preprint doi: https://doi.org/10.1101/701953; this version posted July 13,2019 . The copyright holder for this preprint (which was not certified by peer review) is the author/funder. All rights reserved. No reuse allowed without permission.

25. Kamentsky, L. et al. Improved structure, function and compatibility for CellProfiler: modular high-throughput image analysis software. Bioinformatics 27, 1179-1180 (2011). 